\title{
Schreier split epimorphisms between monoids
}

\author{
Dominique Bourn *, Nelson Martins-Ferreira ${ }^{\dagger}$, \\ Andrea Montoli $\ddagger_{\text {and Manuela Sobral }}^{\S}$
}

January 9, 2014

\begin{abstract}
We explore some properties of Schreier split epimorphisms between monoids, which correspond to monoid actions. In particular, we prove that the split short five lemma holds for monoids, when it is restricted to Schreier split epimorphisms, and that any Schreier reflexive relation is transitive, partially recovering in monoids a classical property of Mal'tsev varieties.
\end{abstract}

Keywords Schreier split epimorphisms, monoids, split short five lemma, internal relations.

\section{Introduction}

Classically, an action of a group $B$ on a group $X$ is defined as a group homomorphism from $B$ to the group $A u t(X)$ of automorphisms of $X$. It is well known that group actions are equivalent to split extensions (which are, in this case, nothing but split epimorphisms). For many other algebraic structures, like Lie algebras, rings, associative algebras and many others, it is still possible to define actions and to obtain the same kind of equivalence with split extensions.

${ }^{*}$ Laboratoire de Mathématiques pures et appliquées, Université du Littoral Côte d'Opale, Calais, France; bourn@lmpa.univ-littoral.fr

${ }^{\dagger}$ ESTG, CDRSP, Instituto Politécnico de Leiria, Leiria, Portugal; martins.ferreira@ipleiria.pt

${ }^{\ddagger}$ CMUC, University of Coimbra, 3001-454 Coimbra, Portugal; montoli@mat.uc.pt

${ }^{\S}$ CMUC and Department of Mathematics, University of Coimbra, 3001-454 Coimbra, Portugal; sobral@mat.uc.pt 
In the case of monoids, actions can be defined in a similar way as for groups: an action of a monoid $B$ on a monoid $X$ can be defined as a monoid homomorphism from $B$ to the monoid $\operatorname{End}(X)$ of endomorphisms of $X$. These actions, however, are not equivalent to split epimorphisms. The question arose, then, to characterize those split epimorphisms that correspond to the actions defined as above. In the paper [6], the authors gave a description of these split epimorphisms, and they called them Schreier split epimorphisms. The name was inspired by the pioneering work of Rédei [8], who introduced the notion of Schreier extension in the context of semigroups, monoids, semirings and semimodules over a semiring, and by the work of Patchkoria [7], who defined the so-called Schreier internal categories in the context of monoids and proved that they are equivalent to what he called crossed semimodules. The definition of crossed semimodule is analogous to the classical one of crossed module of groups, and uses the notion of monoid action described at the beginning. Moreover, Patchkoria's work was generalized in [6] to the case of monoids with operations, a wide class of algebraic structures, which includes monoids, semirings, join-semilattices with a bottom element, distributive lattices with a top (or a bottom) element and other examples.

The aim of the present paper is to start a deep study of Schreier split epimorphisms, showing that they have many interesting properties, typical of all split epimorphisms of groups, that are not valid, in general, for split epimorphisms between monoids. An example is the fact that a Schreier split epimorphism is necessarily the cokernel of its kernel. A first important property is the split short five lemma: given a commutative diagram

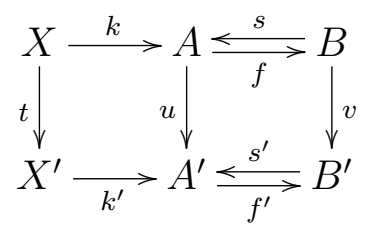

of monoid homomorphisms, where the two rows are Schreier split epimorphisms (together with their kernels), $u$ is an isomorphism if and only if both $t$ and $v$ are. This is not true for general split epimorphisms between monoids, but it is well known to hold in the case of groups. This lemma is a key step in order to construct group cohomology. The fact that it holds for Schreier split epimorphisms between monoids can then be used to develop a cohomology theory for monoids (see [2]).

A second important property studied in the present paper is related to 
Schreier reflexive relations. An internal relation on an algebra $B$ in a variety of universal algebra is nothing but a subalgebra of the product $B \times B$; in other words, it is completely described by a pair of homomorphisms

$$
R \underset{d_{1}}{\stackrel{d_{0}}{\longrightarrow}} B
$$

that are jointly monomorphic. An internal relation is reflexive when $d_{0}$ and $d_{1}$ have a common section $s_{0}$. It is well known that, in Mal'tsev varieties [5], every internal reflexive relation is an equivalence relation. This is false in the variety of monoids. However, this property can be partially recovered if we restrict our attention to Schreier reflexive relations, i.e. internal reflexive relations of the form

$$
R \underset{d_{1}}{\stackrel{d_{0}}{\rightleftarrows s_{0}} \underset{\longrightarrow}{\longrightarrow}} B
$$

such that the split epimorphism $\left(d_{0}, s_{0}\right)$ is a Schreier one, see Theorem 5.5.

The paper is organized as follows. In Section 2 we introduce the notion of Schreier split epimorphism and the stronger one of homogeneous split epimorphism, and we describe their first properties, including the fact that a Schreier split epimorphism is the cokernel of its kernel. In Section 3 we give some examples of Schreier and homogeneous split epimorphisms. In Section 4 we prove that the split short five lemma holds for monoids when it is restricted to Schreier split epimorphisms. In Section 5 we study the Schreier reflexive relations, showing that they have some typical properties of reflexive relations in Mal'tsev varieties.

\section{Definitions and first properties}

Throughout all the paper, a split epimorphism between monoids, i.e. a diagram of the form

$$
A \underset{f}{\stackrel{s}{\leftrightarrows}} B
$$

such that $f s=1_{B}$, will be denoted by a 4 -tuple $(A, B, f, s)$. The kernel of $f$ will be denoted by $K[f]$.

We start by introducing the following definition (which is inspired by the definition of homogeneous internal category given in [4]):

Definition 2.1. A split epimorphism $(A, B, f, s)$ between monoids is said to be right homogeneous when, for any element $b \in B$, the map $\mu_{b}: K[f] \rightarrow$ 
$f^{-1}(b)$ defined by the multiplication on the right by $s(b)$, as $\mu_{b}(k)=k \cdot s(b)$, is bijective. Similarly, by duality, we can define a left homogeneous split epimorphism. ( $A, B, f, s)$ is said to be homogeneous when it is both right and left homogeneous.

The following notion (which was inspired by the notion of Schreier internal category introduced in [7]) first appeared in [6] (Definition 2.6):

Definition 2.2. A split epimorphism $(A, B, f, s)$ between monoids is said to be a Schreier split epimorphism when, for any $a \in A$, there exists a unique $\alpha$ in the kernel $K[f]$ of $f$ such that $a=\alpha \cdot s f(a)$.

In other terms, a Schreier split epimorphism is a split epimorphism $(A, B, f, s)$ equipped with a unique set-theoretical map $q: A \rightarrow K[f]$ with the property that, for any $a \in A$, we have:

$$
a=q(a) \cdot s f(a)
$$

The two notions of right homogeneous and of Schreier split epimorphism are equivalent, as the following proposition shows:

Proposition 2.3. A split epimorphism $(A, B, f, s)$ is right homogeneous if and only if it is a Schreier split epimorphism.

Proof. Given a right homogeneous split epimorphism $(A, B, f, s)$, the unique $\alpha$ that appears in Definition 2.2 is given by $\alpha=\mu_{f(a)}^{-1}(a)$. Conversely, given a Schreier split epimorphism, for any $b \in B$ the inverse of the map $\mu_{b}: K[f] \rightarrow$ $f^{-1}(b)$ is defined in the following way: if $a \in f^{-1}(b), \mu_{b}^{-1}(a)$ is the unique $\alpha \in K[f]$ such that $a=\alpha \cdot s f(a)$.

There is an analogous description of left homogeneous split epimorphisms. We do not give it explicitly, because the description of right homogeneous, and, symmetrically, of left homogeneous split epimorphisms given in the following proposition will be more useful later in the paper.

Proposition 2.4. A split epimorphism $(A, B, f, s)$ is right homogeneous (i.e. a Schreier split epimorphism) if and only if there exists a set-theoretical map $q: A \rightarrow K[f]$ such that:

$$
\begin{aligned}
q(a) \cdot s f(a) & =a \\
q(\alpha \cdot s(b)) & =\alpha
\end{aligned}
$$


for every $a \in A, \alpha \in K[f]$ and $b \in B$. Dually, a split epimorphism is left homogeneous if and only if there exists a set-theoretical map $\bar{q}: A \rightarrow K[f]$ such that:

$$
\begin{aligned}
s f(a) \cdot \bar{q}(a) & =a \\
\bar{q}(s(b) \cdot \alpha) & =\alpha
\end{aligned}
$$

for every $a \in A, \alpha \in K[f]$ and $b \in B$.

Proof. Suppose that for every $a \in A$, there exists a unique $\alpha \in K[f]$ such that $a=\alpha \cdot s f(a)$. This property defines a map $q: A \rightarrow K[f]$, by $q(a)=\alpha$, such that $a=q(a) \cdot s f(a)$, for every $a \in A$. In order to prove that $q(\alpha \cdot s(b))=\alpha$ for any $\alpha \in K[f]$, it suffices to observe that $s f(\alpha \cdot s(b))=s(b)$.

Conversely, given a set-theoretical map $q: A \rightarrow B$ satisfying the asserted identities, we can choose $\alpha=q(a)$ for every $a \in A$ by the first identity; suppose now that $a=\alpha^{\prime} \cdot s f(a)$, then we get:

$$
q(a)=q\left(\alpha^{\prime} \cdot s f(a)\right)=\alpha^{\prime}
$$

by the second identity. The proof for the case of left homogeneous split epimorphisms is similar.

We will call the following diagram:

$$
K[f] \underset{k}{\stackrel{q}{<}} \rightarrow \underset{f}{\stackrel{s}{\longrightarrow}} B,
$$

the canonical Schreier split sequence associated with the Schreier split epimorphism, and $q$ the associated Schreier retraction. We have the following properties:

Lemma 2.5. Given a Schreier split epimorphism $(A, B, f, s)$, we have:

(a) $q k=1_{K[f]}$, i.e. $q k$ is the identity map on $K[f]$;

(b) qs is the null homomorphism, i.e. $q s(b)=1$ for every $b \in B$;

(c) $q(1)=1$.

Proof. (a) is a straighforward consequence of the second identity in Proposition 2.4.

(b) for $b \in B$ we have:

$$
s(b)=1 \cdot s f(s(b))
$$

and the uniqueness of $q$ gives that $q s(b)=1$ for every $b \in B$. 
(c) obviously we have $1=1 \cdot s f(1)$.

Proposition 2.6. If $(A, B, f, s)$ is a Schreier split epimorphism, then $f$ is the cokernel of $k$. In other words the split sequence

$$
1 \longrightarrow K[f] \stackrel{k}{\longrightarrow} A \underset{f}{\stackrel{s}{\rightleftarrows}} B \longrightarrow 1
$$

where 1 is the trivial monoid, is exact.

Proof. Given a homomorphism $g: A \rightarrow D$ such that $g k$ is the null homomorphism, we have that $g s$ makes the triangle below commutative:

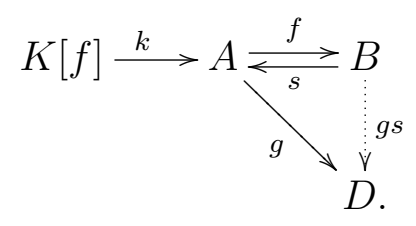

Indeed, since $g k(x)=1$ for any $x \in K[f]$, then $g q(a)=1$ for any $a \in A$. Then, for any $a \in A$, we have:

$$
g(a)=g(q(a) \cdot s f(a))=g q(a) \cdot g s f(a)=1 \cdot g s f(a)=g s f(a),
$$

and hence $g s f=g$. Moreover, given any $h: B \rightarrow D$ such that $h f=g$, we have that

$$
h=h f s=g s .
$$

\section{Examples of Schreier and homogeneous split epimorphisms}

Proposition 3.1. Given any direct product diagram

$$
X \underset{\left\langle 1_{X}, 0\right\rangle}{\stackrel{\pi_{X}}{\rightleftarrows}} X \times B \underset{\left\langle 0,1_{B}\right\rangle}{\stackrel{\pi_{B}}{\rightleftarrows}} B
$$

the canonical split epimorphism $\left(X \times B, B, \pi_{B},\left\langle 0,1_{B}\right\rangle\right)$ is homogeneous.

Proof. The equality $(x, 1) \cdot(1, b)=(x, b)$ shows that it is right homogeneous, while $(1, b) \cdot(x, 1)=(x, b)$ shows that it is left homogeneous. Here the Schreier retraction $\pi_{X}$ is a monoid homomorphism. 
Corollary 3.2. The split epimorphism

$$
X \rightleftarrows 1
$$

is homogeneous.

Corollary 3.3. The identity split epimorphism

$$
X \underset{1_{X}}{\stackrel{1_{X}}{\rightleftarrows}} X
$$

and more generally any isomorphism, is homogeneous.

Proposition 3.4. If $B$ is a group, then every split epimorphism $(A, B, f, s)$ is homogeneous.

Proof. Given a split sequence of the form

$$
K[f] \stackrel{k}{\longrightarrow} A \underset{f}{\stackrel{s}{\longrightarrow}} B
$$

let us define, for any $a \in A, q(a)=a \cdot s f(a)^{-1} \in K[f]$. This map $q$ clearly satisfies the conditions of Proposition 2.4, showing that it is a Schreier split epimorphism. The map $\bar{q}(a)=s f(a)^{-1} \cdot a \in K[f]$, which satisfies the dual conditions, shows that it is left homogeneous.

We will prove later that the converse is also true: if any split epimorphism with codomain $B$ is homogeneous, then $B$ is a group.

Example 3.5. Consider the internal order in the variety of monoids given by the usual order between natural numbers:

$$
\mathcal{O}_{\mathbb{N}} \underset{p_{1}}{\stackrel{p_{0}}{<s_{0}} \underset{\longrightarrow}{\longrightarrow}} \mathbb{N}
$$

where

$$
\mathcal{O}_{\mathbb{N}}=\{(x, y) \in \mathbb{N} \times \mathbb{N} \mid x \leq y\},
$$

$p_{0}$ and $p_{1}$ are the first and the second projection, and $s_{0}(x)=(x, x)$. Then the split epimorphism $\left(\mathcal{O}_{\mathbb{N}}, \mathbb{N}, p_{0}, s_{0}\right)$ is homogeneous.

Proof. The equality $(x, y)=(0, y-x)+(x, x)$, for any $(x, y) \in \mathcal{O}_{\mathbb{N}}$ proves that the split epimorphism in question is right homogeneous, while the commutativity implies that it is left homogeneous. 
Example 3.6. We denote by $\mathbb{Z}^{*}$ the monoid of non-zero integers with the usual multiplication, and by $\mathbb{N}^{*}$ its submonoid whose elements are the numbers greater than 0 . Then the split epimorphism

$$
\mathbb{Z}^{*} \underset{a b s}{\stackrel{i}{\rightleftarrows}} \mathbb{N}^{*}
$$

where $i$ is the inclusion and abs associates with any integer its absolute value, is a homogeneous split epimorphism. In fact $K[a b s]=\{ \pm 1\}$, and it is immediate to see that any non-zero integer $z$ can be written in a unique way as $z= \pm 1 \cdot|z|=|z| \cdot \pm 1$.

Example 3.7. Let us fix a natural number $n$. We can define on the cartesian product $\mathbb{N} \times \mathbb{N}$ the monoid structure given by

$$
\left(x_{1}, b_{1}\right) \cdot\left(x_{2}, b_{2}\right)=\left(x_{1}+n^{b_{1}} x_{2}, b_{1}+b_{2}\right) .
$$

(when both $n$ and $b_{1}$ are 0 , we use the convention $0^{0}=1$ ). We denote this monoid by $\mathbb{N} \rtimes_{n} \mathbb{N}$. The projection $\pi_{1}: \mathbb{N} \rtimes_{n} \mathbb{N} \rightarrow \mathbb{N}$ defined by $\pi_{1}(x, b)=b$ is a monoid homomorphism, in the same way as the section $\sigma_{1}: \mathbb{N} \rightarrow \mathbb{N} \rtimes_{n} \mathbb{N}$ defined by $\sigma_{1}(b)=(0, b)$. For any $n \in \mathbb{N}$, the split epimorphism $\left(\mathbb{N} \rtimes_{n}\right.$ $\mathbb{N}, \mathbb{N}, \pi_{1}, \sigma_{1}$ ) is a Schreier split epimorphism: the (unique) map $q: \mathbb{N} \rtimes_{n} \mathbb{N} \rightarrow \rightarrow$ $K\left[\pi_{1}\right]$ is just the first projection $\pi_{0}: \mathbb{N} \times \mathbb{N} \rightarrow \mathbb{N}$. If $n=0$, this split epimorphism is not homogeneous (because it is not left homogeneous): for example, for $b=1$, the map $\lambda_{1}: K\left[\pi_{1}\right] \rightarrow \pi_{1}^{-1}(1)$ defined by

$$
\lambda_{1}(x, 0)=(0,1) \cdot(x, 0)=(0,1)
$$

is clearly not bijective.

The last example is an application of the fact, proved in [6], that Schreier split epimorphisms correspond to monoid actions. Let us briefly recall the equivalence between these two concepts. Given a Schreier split epimorphism

$$
K[f] \underset{k}{\stackrel{q}{<}} \rightarrow \underset{f}{\stackrel{s}{\longleftarrow}} B
$$

we obtain an action of $B$ on $K[f]$, i.e. a monoid homomorphism $\varphi: B \rightarrow$ $\operatorname{End}(K[f])$, by putting

$$
\varphi(b)(x)=q(s(b) \cdot x)
$$

for any $b \in B$ and any $x \in K[f]$. Conversely, given a homomorphism $\psi: B \rightarrow$ $\operatorname{End}(X)$, we can define, on the cartesian product $X \times B$, a binary operation as follows:

$$
\left(x_{1}, b_{1}\right) \cdot\left(x_{2}, b_{2}\right)=\left(x_{1} \cdot \psi\left(b_{1}\right)\left(x_{2}\right), b_{1} \cdot b_{2}\right) .
$$


It is easy to see that, in this way, we obtain a monoid, denoted by $X \rtimes_{\psi} B$, and a Schreier split epimorphism

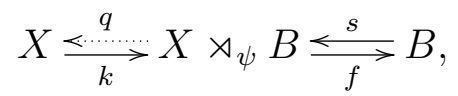

where $f$ and $q$ are the canonical projections of the product, while $k$ and $s$ are the inclusions of $X$ and $B$, respectively (this construction is inspired by the classical semidirect product of groups).

In particular, the Schreier split epimorphisms having $\mathbb{N}$ both as codomain and kernel are in bijection with the monoid homomorphisms $\mathbb{N} \rightarrow \operatorname{End}(\mathbb{N})$. Hence it is easy to see that all such Schreier split epimorphisms are obtained, up to isomorphisms, by the construction (1).

We have a similar description for homogeneous split epimorphisms: the homogeneous split epimorphisms with codomain $B$ and kernel $X$ correspond to the monoid homomorphisms $B \rightarrow \operatorname{Aut}(X)$, where $\operatorname{Aut}(X)$ is the group of automorphisms of $X$, as the following proposition shows.

Proposition 3.8. A Schreier split epimorphism

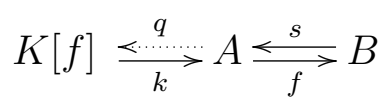

is homogeneous if and only if the corresponding monoid homomorphism $\varphi: B \rightarrow \operatorname{End}(K[f])$ factors through Aut $(K[f])$.

Proof. Suppose that $(A, B, f, s)$ is homogeneous. Let us show that any endomorphism $\varphi(b)$ is an automorphism. Suppose $q(s(b) \cdot x)=q\left(s(b) \cdot x^{\prime}\right)$, with $b \in B$ and $x, x^{\prime} \in K[f]$. Then we have

$s(b) \cdot x=q(s(b) \cdot x) \cdot s f(s(b) \cdot x)=q(s(b) \cdot x) \cdot s(b)=q\left(s(b) \cdot x^{\prime}\right) \cdot s(b)=s(b) \cdot x^{\prime}$.

Since the split epimorphism is left homogeneous, we have $x=x^{\prime}$ and $\varphi(b)$ is injective. Let $z$ be in $K[f]$. Since the split epimorphism is left homogeneous, there exists $t \in K[f]$ such that $s(b) \cdot t=z \cdot s(b)$. Then

$$
\varphi(b)(t)=q(s(b) \cdot t)=q(z \cdot s(b))=z
$$

since $z \in K[f]$, and consequently $\varphi(b)$ is surjective. Conversely, suppose that the Schreier split epimorphism $(A, B, f, s)$ corresponds to a homomorphism 
$\varphi: B \rightarrow A u t(K[f])$. This means that $(A, B, f, s)$ is isomorphic to the Schreier split epimorphism

$$
K[f] \underset{\left\langle 1_{K[f]}, 0\right\rangle}{\stackrel{\pi_{K[f]}}{\longrightarrow}} K[f] \rtimes_{\varphi} B \underset{\pi_{B}}{\stackrel{\left\langle 0,1_{B}\right\rangle}{\rightleftharpoons}} B
$$

We have to show that, for any $b \in B$, the map $\mu_{b}: K[f] \rightarrow \pi_{B}^{-1}(b)$ defined by

$$
\mu_{b}(x)=(1, b) \cdot(x, 1)=(\varphi(b)(x), b)
$$

is bijective, but this follows immediately from the fact that $\varphi(b)$ is an automorphism.

\section{The Schreier split short five lemma}

The aim of this section is to prove that the split short five lemma holds also for monoids, if we restrict our attention to Schreier split epimorphisms. We will need the following auxiliary result:

Lemma 4.1. Consider the following commutative diagram, where the two rows are Schreier split epimorphisms:

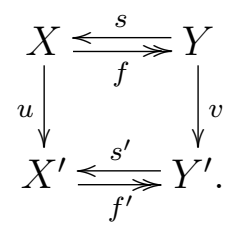

Then, in the following diagram, completed with the kernels and the restriction $K(u)$ of $u$, the leftward left hand side square commutes:

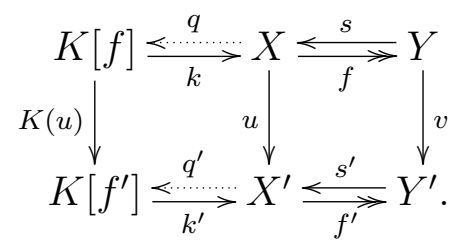

In other terms, we have that $q^{\prime} u=K(u) q$.

Proof. We have to show that $q^{\prime} u(x)=u q(x)$ for any $x$ in the monoid $X$. It is true since we have:

$u q(x) \cdot s^{\prime} f^{\prime} u(x)=u q(x) \cdot u s f(x)=u(q(x) \cdot s f(x))=u(x)=q^{\prime} u(x) \cdot s^{\prime} f^{\prime} u(x)$

and then the thesis follows from the uniqueness in the Schreier condition. 
Theorem 4.2. Consider the following commutative diagram, where the two rows are Schreier split sequences and $K(u)$ is the restriction of $u$ to the kernels:

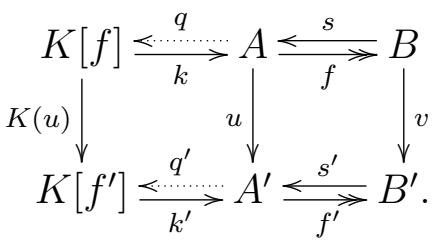

We have that

(i) $u$ is a surjective homomorphism if and only if both $v$ and $K(u)$ are;

(ii) $u$ is a monomorphism if and only if both $v$ and $K(u)$ are;

(iii) $u$ is an isomorphism if and only if both $v$ and $K(u)$ are.

Proof. (i) If $u$ is a surjective homomorphism, then so is $v f=f^{\prime} u$, and this implies that $v$ is surjective. The map $q^{\prime}$ is surjective (this is an immediate consequence of Lemma 2.5 (a)), then so is $q^{\prime} u=K(u) q$, and this implies that $K(u)$ is surjective. Conversely, suppose that $v$ and $K(u)$ are surjective. Consider $a^{\prime} \in A^{\prime}$. There are $\alpha \in K[f]$ and $b \in B$ such that $u(\alpha)=q^{\prime}\left(a^{\prime}\right)$ and $v(b)=f^{\prime}\left(a^{\prime}\right)$. Accordingly

$$
u(\alpha \cdot s(b))=u(\alpha) \cdot u s(b)=q^{\prime}\left(a^{\prime}\right) \cdot s^{\prime} v(b)=q^{\prime}\left(a^{\prime}\right) \cdot s^{\prime} f^{\prime}\left(a^{\prime}\right)=a^{\prime} .
$$

Hence the homomorphism $u$ is surjective.

(ii) If $u$ is a monomorphism, then so is $u s=s^{\prime} v$, which implies that $v$ is a monomorphism. Similarly $u k=k^{\prime} K(u)$ is a monomorphism, which implies that $K(u)$ is a monomorphism. Conversely, suppose that $K(u)$ and $v$ are monomorphisms. Suppose now that $u\left(a_{1}\right)=u\left(a_{2}\right)$. Then we have $f^{\prime} u\left(a_{1}\right)=f^{\prime} u\left(a_{2}\right)$ and then $v f\left(a_{1}\right)=v f\left(a_{2}\right)$, whence $f\left(a_{1}\right)=$ $f\left(a_{2}\right)$. Moreover, from $u\left(a_{1}\right)=u\left(a_{2}\right)$, we can conclude that

$$
K(u) q\left(a_{1}\right)=q^{\prime} u\left(a_{1}\right)=q^{\prime} u\left(a_{2}\right)=K(u) q\left(a_{2}\right) .
$$

Since the restriction $K(u)$ of $u$ to $K[f]$ is a monomorphism, we get $q\left(a_{1}\right)=q\left(a_{2}\right)$. With $f\left(a_{1}\right)=f\left(a_{2}\right)$, we get $a_{1}=a_{2}$.

(iii) It follows immediately from (i) and (ii). 


\section{$5 \quad$ Schreier internal relations}

As we recalled in the introduction, by internal relation on an algebra $B$ in a variety of universal algebra we mean a subalgebra of the product $B \times B$. By considering the homomorphic inclusion

$$
R \longmapsto B \times B
$$

and by composing it with the two projections of the product, we get two parallel homomorphisms

$$
R \underset{d_{1}}{\stackrel{d_{0}}{\longrightarrow}} B,
$$

that are the first and the second projection of the relation. More explicitly, denoting an element of $R$ by a pair $x R y$, such that $x$ and $y$ belong to $B$ and are linked by the relation $R$, we have that $d_{0}(x R y)=x$ and $d_{1}(x R y)=y$.

An internal relation is reflexive when $d_{0}$ and $d_{1}$ have a common section $s_{0}: B \rightarrow R$. In the notation above, we have that $s_{0}(b)=b R b$ for any $b \in B$. A fundamental property of Mal'tsev varieties [5] is that every internal reflexive relation is an equivalence relation. In the variety of monoids this is not true. For instance, the relations of Examples 5.3 and 5.4 below are internal reflexive relations which are not symmetric. However, we are going to show in this section that the property mentioned above can be partially recovered if we restrict our attention to the Schreier reflexive relations, whose definition is the following.

Definition 5.1. An internal reflexive relation of monoids

$$
R \underset{d_{1}}{\stackrel{d_{0}}{\rightleftarrows s_{0}}} \underset{\longrightarrow}{\longrightarrow} B
$$

is a Schreier reflexive relation if the split epimorphism $\left(R, B, d_{0}, s_{0}\right)$ is a Schreier one. It is homogeneous if $\left(R, B, d_{0}, s_{0}\right)$ is homogeneous.

Example 5.2. For every monoid $X$, the discrete internal equivalence relation:

$$
X \underset{1_{X}}{\stackrel{1_{X}}{\underset{1_{X}}{\longrightarrow}}} X,
$$

i.e. the relation $R$ such that $x R y$ if and only if $x=y$, is a homogeneous internal equivalence relation. 
Example 5.3. Example 3.5 shows that the internal order in the variety of monoids given by the usual order between natural numbers:

$$
\mathcal{O}_{\mathbb{N}} \underset{p_{1}}{\stackrel{p_{0}}{<s_{0}} \underset{\longrightarrow}{\longrightarrow}} \mathbb{N}
$$

where

$$
\mathcal{O}_{\mathbb{N}}=\{(x, y) \in \mathbb{N} \times \mathbb{N} \mid x \leq y\},
$$

is a homogeneous order relation.

Example 5.4. Since $\mathbb{Z}$, with the usual sum, is a group, the internal order in monoids given by the usual order between integers:

$$
\mathcal{O}_{\mathbb{Z}} \underset{p_{1}}{\stackrel{p_{0}}{\underset{s_{0}}{\longrightarrow}}} \mathbb{Z},
$$

where

$$
\mathcal{O}_{\mathbb{Z}}=\{(x, y) \in \mathbb{Z} \times \mathbb{Z} \mid x \leq y\},
$$

is a homogeneous order relation (thanks to Proposition 3.4).

The previous example can be obviously generalized to the case of the order relation $\mathcal{O}_{G}$ associated with any ordered group $G$.

We are now ready to prove the main result of this section. We will use the fact that every Schreier reflexive relation is right homogeneous (because of Proposition 2.3).

Theorem 5.5. Any Schreier reflexive relation is transitive. It is an equivalence relation if and only if $K\left[d_{0}\right]$ is a group.

Proof. Let us first observe that the elements of $K\left[d_{0}\right]$ are of the form $1 R b$, with $b \in B$.

Suppose that $x R y$ and $y R z$. Since the reflexive relation is a Schreier one, and hence right homogeneous, from $x R y$ we know that there is a unique element $t \in B$ such that $1 R t$ and

$$
x R y=(1 R t) \cdot s_{0}(x)=(1 R t) \cdot(x R x)=(1 \cdot x) R(t \cdot x),
$$

which gives $t \cdot x=y$; from $y R z$ we get an element $\tau \in B$ such that $1 R \tau$ and

$$
y R z=(1 R \tau) \cdot s_{0}(y)=(1 R \tau) \cdot(y R y)=(1 \cdot y) R(\tau \cdot y),
$$

which gives $\tau \cdot y=z$. Hence $1 R(\tau \cdot t)$ and then $x R(\tau \cdot t \cdot x)=x R(\tau \cdot y)=x R z$. 
Suppose now that $R$ is symmetric. Then any $y$ such that $1 R y$ is such that $y R 1$. Since $R$ is a Schreier reflexive relation, there is a unique element $y^{\prime}$ such that $1 R y^{\prime}$ and

$$
y R 1=1 R y^{\prime} \cdot s_{0}(y)=1 R y^{\prime} \cdot y R y=(1 \cdot y) R\left(y^{\prime} \cdot y\right),
$$

which gives $y^{\prime} \cdot y=1$; so, any element $y \in K\left[d_{0}\right]$ has a left inverse, which implies that $K\left[d_{0}\right]$ is a group. Conversely, suppose that $K\left[d_{0}\right]$ is a group and that we have $x R y$. There is an element $t$ such that $1 R t$ and

$$
x R y=(1 R t) \cdot s_{0}(x)=(1 R t) \cdot(x R x)=(1 \cdot x) R(t \cdot x),
$$

which gives $t \cdot x=y$. There exists $t^{-1}$ such that $1 R t^{-1}$. Whence $y R\left(t^{-1} \cdot y\right)=$ $y R x$.

Examples 5.3 and 5.4 are instances of Schreier reflexive relations that are not equivalence relations; actually they are order relations.

Proposition 5.6. Given a monoid $X$, the indiscrete equivalence relation $\nabla_{X}$ given by:

$$
X \times X \underset{p_{1}}{\stackrel{p_{0}}{\underset{s_{0}}{\longrightarrow}}} X,
$$

i.e. the relation $R$ such that $x R y$ for any $x, y \in X$, is a Schreier equivalence relation if and only if $X$ is a group. In this case $\nabla_{X}$ is actually homogeneous.

Proof. If $X$ is a group, it is an immediate consequence of Proposition 3.4; actually the equivalence relation $\nabla_{X}$ is even homogeneous. Conversely, suppose the indiscrete equivalence relation is a Schreier equivalence relation. Then, according to the previous proposition, $K\left[p_{0}\right]=X$ is a group.

We can now prove the converse of Proposition 3.4:

Corollary 5.7. Given any monoid B, the three following conditions are equivalent:

(a) the monoid $B$ is a group

(b) any split epimorphism with codomain $B$ is homogeneous

(c) any split epimorphism with codomain $B$ is a Schreier split epimorphism.

Proof. $(a) \Rightarrow(b)$ is given by Proposition 3.4.

$(b) \Rightarrow(c)$ holds since any homogeneous split epimorphism is a Schreier one.

$(c) \Rightarrow(a)$ Condition (c) implies that the indiscrete relation $\nabla_{B}$ is a Schreier equivalence relation, and consequently $B$ is a group. 


\section{Conclusion}

In this paper we showed some important properties of Schreier split epimorphisms between monoids. They gave evidence to the need of a more systematic study of these structures, which will allow to understand better the intrinsic properties of monoids, as it was already done for groups and other structures, like rings and Lie algebras, with the notions of protomodular [1] and Mal'tsev [3] categories. This systematic study, together with an extension of the results presented in this paper to the case of semirings, will appear in the forthcoming work [2], in which, in particular, some cohomological properties are investigated.

\section{Acknowledgements}

This work was partially supported by the Centro de Matemática da Universidade de Coimbra (CMUC), funded by the European Regional Development Fund through the program COMPETE and by the Portuguese Government through the FCT - Fundação para a Ciência e a Tecnologia under the project PEst-C/MAT/UI0324/2013 and grants number PTDC/MAT/120222/2010 and SFRH/BPD/69661/2010, and also by ESTG and CDRSP from the Polytechnical Institute of Leiria.

\section{References}

[1] D. Bourn, Normalization equivalence, kernel equivalence and affine categories, in Lecture Notes in Mathematics, vol. 1488 (1991), SpringerVerlag, 43-62.

[2] D. Bourn, N. Martins-Ferreira, A. Montoli, M. Sobral, Schreier split epimorphisms in monoids and in semirings, Textos de Matemática Série B, Departamento de Matemática da Universidade de Coimbra, vol. 45 (2014).

[3] A. Carboni, J. Lambek, M.C. Pedicchio, Diagram chasing in Mal'cev categories, J. Pure Appl. Algebra 69 (1990), 271-284.

[4] R. Lavendhomme, J.R. Roisin, Cohomologie non abélienne de structures algébriques, J. Algebra 67 (1980), 385-414.

[5] A. I. Mal'cev, On the general theory of algebraic systems, Mat. Sbornik N. S. 35 (1954), 3-20. 
[6] N. Martins-Ferreira, A. Montoli, M. Sobral, Semidirect products and crossed modules in monoids with operations, J. Pure Appl. Algebra 217 (2013), 334-347.

[7] A. Patchkoria, Crossed semimodules and Schreier internal categories in the category of monoids, Georgian Math. Journal 5 n.6 (1998), 575-581.

[8] L. Rédei, Die Verallgemeinerung der Schreierschen Erweiterungstheorie, Acta Sci. Math. Szeged 14 (1952), 252-273. 